Rev. Elet. em Gestão, Educação e Tecnologia Ambiental (e-ISSN: 2236-1170)

\title{
AVALIAÇÃO DA SUSTENTABILIDADE AMBIENTAL EM BACIAS HIDROGRÁFICAS MEDIANTE A APLICAÇÃO DO MODELO P-E-R
}

\author{
Pedro Daniel da Cunha Kemerich', Sergio Roberto Martins², Masato Kobyama³ , Galileo Adeli \\ Buriol $^{4}$, Willian Fernando de Borba ${ }^{5}$, Luciana Gregory Ritter ${ }^{6}$
}

\begin{abstract}
${ }^{1}$ Graduação em Engenharia Ambiental pelo Centro Universitário Franciscano -UNIFRA (2005). Mestrado pela Universidade Federal de Santa Maria - UFSM, em Recursos Hídricos e Saneamento Ambiental (2008), Pós-Graduação em Engenharia de Segurança do Trabalho pelo Centro Universitário Franciscano - UNIFRA (2009) e é Doutorando do Programa de Pós-Graduação em Engenharia Ambiental da Universidade Federal de Santa Catarina. É Coordenador do Curso de Engenharia Ambiental da Universidade Federal de Santa Maria/CESNORS. pdck@pop.com.br

${ }^{2}$ Graduação e mestrado em Agronomia pela Universidade Federal de Pelotas, doutorado e pós-doutorado em Agronomia pela Universidade Politécnica de Madrid, mestrado em Gestão Econômica e Planejamento do Desenvolvimento pela Universidad Complutense de Madrid. Professor Visitante Nacional Senior/CAPES da Universidade Federal da Fronteira Sul. Professor Colaborador do Programa de Pós-Graduação em Engenharia Ambiental PPGEA/UFSC. sergio@ens.ufsc.br

${ }^{3}$ Graduação em Ciências Especiais pela Kyoto Educational University, mestrado em Forestry Science pela Tokyo University of Agriculture and Technology e doutorado em Engenharia Florestal pela Universidade Federal do Paraná.

Professor Associado III da Universidade Federal de Santa Catarina, Programa de Pós-Graduação em Engenharia Ambiental - PPGEA/UFSC. kobiyama@ens.ufsc.br

4Graduação e Mestrado em Agronomia pela Universidade Federal de Santa Maria, doutorado em Bioclimatologia

Vegetal pela Universite de Paris VII - Universite Denis Diderot, Docteur Ingenier. Professor Titular do Centro Universitário Franciscano - UNIFRA. galileo@unifra.br

5Técnico em Agropecuária pela Universidade Federal de Santa Maria (2007). Aluno de Graduação do Curso de Engenharia Ambiental da Universidade Federal de Santa Maria (2009 - 2014). Trabalha nas áreas de contaminação de solo, resíduos sólidos, águas subterrâneas e recursos hídricos. borba willian@hotmail.com 6Aluna de Graduação do Curso de Engenharia Ambiental da Universidade Federal de Santa Maria. lucianaritter@yahoo.com
\end{abstract}

http://dx.doi.org/10.5902/223611707658

\section{RESUMO}

Um instrumento fundamental para o gerenciamento de bacias hidrográficas é o conhecimento dos indicadores de sustentabilidade, descrevendo-se a dinâmica dos problemas ambientais existentes na área de interesse. Faz-se necessário identificar os indicadores de: Pressão que traduzem a utilização dos recursos naturais (solo, água e ar) e dos fatores de produção (fertilizantes, produtos fitofarmacêuticos e energia); Estado que avaliam os impactos sobre o solo, a água, o ar, a biodiversidade, os habitats e a paisagem; Resposta que expressam os esforços sociais e políticos que são desenvolvidos no sentido de melhorar o desempenho ambiental. O presente trabalho teve como objetivo demonstrar o uso de indicadores de sustentabilidade ambiental aliado à análise de vulnerabilidade natural à contaminação da água subterrânea em bacia hidrográfica. Com base no objetivo proposto, pode-se concluir que os indicadores de sustentabilidade aliados à aplicação de metodologias de análise de vulnerabilidade são capazes de identificar quantificar os impactos ambientais em bacias hidrográficas, especialmente em suas áreas mais susceptíveis a eventos danosos ao meio ambiente. Dessa forma é possível também identificar as medidas tomadas até o momento da avaliação e incrementá-las e/ou modificá-las tornando-as mais 
Rev. Elet. em Gestão, Educação e Tecnologia Ambiental (e-ISSN: 2236-1170)

eficientes podendo estas serem direcionadas as áreas onde realmente se fazem necessárias, demonstrando assim a importância de tais instrumentos no gerenciamento de recursos naturais.

Palavras-chave: Pressão, estado, resposta, gerenciamento de bacias hidrográficas.

\begin{abstract}
A key tool for watershed management is knowledge of sustainability indicators, describing the dynamics of environmental problems in the area of interest. It is necessary to identify indicators: Pressure that reflect the use of natural resources (soil, water and air) and the factors of production (fertilizers, pesticides and energy) state that assess the impacts on soil, water, air, biodiversity, habitats and landscapes; Answer expressing social and political efforts that are designed to improve environmental performance. The present study aimed to demonstrate the use of indicators of environmental sustainability combined with the analysis of natural vulnerability to groundwater contamination in the watershed. Based on the proposed objective, it can be concluded that indicators of sustainability combined with the application of methodologies for vulnerability analysis can identify quantify environmental impacts on watersheds, especially in their areas more susceptible to events harmful to the environment. So you can also identify the measures taken to date assessment and enhance them and / or modify them to make them more efficient can be directed to those areas where they really are needed, thus demonstrating the importance of such tools in managing natural resources.
\end{abstract}

Keywords: pressure, state, response, watershed management.

\title{
INTRODUÇÃO
}

A bacia hidrográfica é adotada como unidade básica de planejamento e gestão de recursos hídricos. O gerenciamento das bacias hidrográficas no Estado do Rio Grande do Sul é realizado pelos Comitês de Gerenciamento de Bacias Hidrográficas. São colegiados instituídos pelo Governo do Estado, integrantes do Sistema Estadual de Recursos hídricos, Lei no 10.350, de 1994. Os representantes dos comitês têm como função discutir e deliberar sobre temas de interesse aos usuários da água, definindo as prioridades do seu uso e as intervenções necessárias à gestão integrada da bacia hidrográfica.

Um instrumento fundamental para o gerenciamento de bacias hidrográficas é o conhecimento dos indicadores de sustentabilidade, descrevendo-se a dinâmica dos problemas ambientais existentes na área de interesse. Faz-se necessário identificar os indicadores de: Pressão que traduzem a utilização dos recursos naturais (solo, água e ar) e dos fatores de produção (fertilizantes, produtos fitofarmacêuticos e energia); Estado que avaliam os impactos sobre o solo, a água, o ar, a biodiversidade, os habitats e a paisagem; Resposta que expressam os esforços sociais e políticos que são desenvolvidos no sentido de melhorar o desempenho ambiental.

Frente à importância de se conhecer os processos de inter-relação entre os componentes ambientais na bacia hidrográfica, é necessária a quantificação dos elementos físicos que a compõem, como orografia, cobertura vegetal, solos, rede de drenagem e balanço hídrico (Beltrame, 1994). No balanço hídrico, o principal componente de entrada é a chuva. Desta forma, a sua determinação temporal e espacial na área da bacia hidrográfica é de fundamental importância para qualquer tomada de decisão que envolva o uso da água. Outro parâmetro 
Rev. Elet. em Gestão, Educação e Tecnologia Ambiental (e-ISSN: 2236-1170)

importante a ser determinado na área de uma bacia hidrográfica são os locais de infiltração máxima de água das chuvas. São áreas em que, em função da natureza do solo e do substrato geológico e, ainda, da morfologia do terreno, possuem condições favoráveis à infiltração da água da chuva, que vão alimentar o lençol freático, podendo também ser áreas vulneráveis a contaminação de origem antrópica. Nesse contexto o presente trabalho teve como objetivo demonstrar o uso de indicadores de sustentabilidade ambiental aliado à análise de vulnerabilidade natural à contaminação da água subterrânea em bacia hidrográfica.

\section{O uso de indicadores de sustentabilidade ambiental}

Os indicadores ambientais começaram a ser utilizados durante a década de 70 e 80, como resultado de esforços de governos e organizações internacionais na elaboração e divulgação dos primeiros Relatórios sobre o Estado do Ambiente, (Franca, 2001). Sendo o governo holandês o pioneiro na adoção de indicadores ambientais, em 1989, para avaliar os resultados da implementação do Plano de Política Ambiental Nacional (Hammond, 1995).

Durante a última década, particularmente em sua segunda metade, desenvolveu-se o interesse na busca de indicadores de sustentabilidade por parte de organismos governamentais, não-governamentais, institutos de pesquisa e universidades em todo o mundo. Muitas conferências já foram organizadas por entidades internacionais, bem como outras iniciativas de pesquisadores ligados a algumas instituições governamentais e/ou universitárias. No entanto, ainda pouco se tem de concreto, pois o tema é relativamente novo para a comunidade acadêmica. Poucas são, também, as publicações que abordam o tema. Muitas informações são obtidas através de conferências eletrônicas que dão indicações sobre os trabalhos que estão sendo desenvolvidos, assim como sobre o direcionamento que vem sendo dado. Os conceitos são novos e os resultados de pesquisa e experimentação, em grande parte dos casos, não estão ainda disponíveis, pois os trabalhos, em sua maioria, estão em andamento (Marzall; Almeida, 2000).

O World Resources Institute (WRI) (Hammond, 1995) fez um levantamento e acompanhou trabalhos que foram ou estão sendo realizados com indicadores de sustentabilidade, principalmente no que se refere a informações ambientais, observando a evolução e o incremento de trabalhos nessa direção nos últimos seis anos. O evento de referência é a Conferência Mundial sobre o Meio Ambiente (Rio-92), com a elaboração de seu documento final, a Agenda 21. Nesse documento, em seu capítulo 40, é enfatizada a necessidade do desenvolvimento de indicadores por parte de cada país, em função de sua realidade. O texto referido cita ainda o Encontro sobre Indicadores Ambientais e de Desenvolvimento Sustentável, em fins de 1993, em Genebra, organizado pelo United Nations Environmental Program (UNEP). Hammond (1995) citam ainda o trabalho desenvolvido pelo governo holandês, que desde 1991 tem o programa de indicadores ambientais que permite o monitoramento efetivo das ações de desenvolvimento, agilizando a tomada de decisões e tendo uma grande participação popular no processo. Pode-se mencionar ainda, no contexto europeu, o colóquio internacional tendo como tema "Indicadores de Desenvolvimento Sustentável", realizado em 1996, na França (Bouni, 1996).

Em 1991, na Áustria, iniciou-se um programa de "eco-pontos", dentro do programa nacional agroambiental, com a aplicação de 13 indicadores, subdivididos em sete para superfícies cultivadas e seis para pastagens. Em 1992 foram desenvolvidas pela Organização Internacional da Luta Biológica e Integrada, na Suíça, experiências-piloto em produção integrada (PI), onde se determinaram as exigências mínimas quanto à $\mathrm{PI}$, e na qual são usados 16 indicadores. $\mathrm{Na}$ 
Rev. Elet. em Gestão, Educação e Tecnologia Ambiental (e-ISSN: 2236-1170)

Alemanha, a Universidade de Bonn desenvolve o diagnóstico biótico, com objetivo de avaliar a biodiversidade (Clain, 1997).

Além desses trabalhos e iniciativas, encontram-se disponíveis on line, via Internet, documentos desenvolvidos por alguns municípios, como o de Rushmoor, na Inglaterra (Macdonalds, 1996), que desenvolvem sua própria Agenda 21, com seus respectivos indicadores, urbanos em sua maioria. Existem ainda estudos efetuados em períodos anteriores que propõem tecnologias para avaliar desempenhos na agricultura. Mas nem sempre é possível interpretá-los à luz dos novos conceitos trazidos pela discussão da sustentabilidade, pois estes representam um referencial maior, requerendo dos especialistas a ultrapassagem de linhas disciplinares bem estabelecidas, com o objetivo de desenvolver trabalhos multi e interdisciplinares (Freyenberg et al, 1996). Além disso, deve-se considerar que, por estarem sendo desenvolvidos em um quadro conceitual e metodológico ainda não totalmente claro, há grande dificuldade para se chegar a um consenso (Marzall, Almeida, 1998).

\section{A função dos indicadores na avaliação da sustentabilidade ambiental}

Os indicadores de sustentabilidade são ferramentas utilizadas para auxiliar no monitoramento da operacionalização do desenvolvimento sustentável, sendo a sua principal função fornecer informações sobre o estado das diversas dimensões (ambientais, econômicas, socioeconômicas, culturais, institucionais, etc.) que compõem o desenvolvimento sustentável do sistema na sociedade (Carvalho, J. et al., 2011).

A utilização de indicadores tem por objetivo reunir e quantificar informações de um modo que sua importância se destaque, simplificando informações sobre fenômenos complexos tentando melhorar com isso o processo de comunicação (Van Bellen, 2006).

Os indicadores exercem uma função fundamental na geração de dados para a avaliação de sustentabilidade, indicando a direção, a prioridade das mudanças e direcionando um caminho de proposta para contribuir com um desenvolvimento sustentável baseados nos agroecossistemas. Sendo assim, um estudo com indicadores não apenas proporciona a construção de propostas de agroecossistemas mais adequados, através da transformação de dados em relevantes informações, mas também informações para a construção de estratégias políticas e de planejamento para um desenvolvimento sustentável.

Através da utilização de indicadores ambientais deve ser possível a análise das condições, mudanças da qualidade ambiental, além de favorecer o entendimento das interfaces da sustentabilidade, bem como de tendências, como uma ferramenta de suporte no processo de tomada de decisão e formulação de políticas e práticas sustentáveis (Gomes; Malheiros, 2012), pois os indicadores têm como função diagnosticar a saúde do ecossistema e fornecer uma ferramenta para monitorar condições e mudanças ambientais ao longo do tempo (Jorgensen, 2005).

A maioria das propostas de avaliação de sustentabilidade são esforços para integrar indicadores, com o objetivo de visualizar o que está ocorrendo em um determinado sistema.

Aprofundando esta teoria e dando origem a estudo de agrupamento de indicadores, Freudenberg (2003) classifica e define os indicadores como: a) Individuais - como sendo uma forma de menu, com indicadores isolados ou um banco de dados estatísticos; b) Temáticos - como indicadores individuais que são agrupados em conjunto considerando suas características acerca 
Rev. Elet. em Gestão, Educação e Tecnologia Ambiental (e-ISSN: 2236-1170)

de um específico tema ou área. Este modelo de avaliação requer uma identificação de determinados tipos de indicadores que estão ligados ou relacionados de alguma forma específica.

Geralmente, esta proposta apresenta os indicadores isoladamente, ao invés de sintetizado em um indicador composto; c) Compostos - formados quando os indicadores temáticos são sintetizados em um índice e apresentados como uma média composta simples.

Segundo Hammond (1995) os indicadores podem informar uma determinada situação, mas também podem passar a ideia de uma percepção de uma tendência ou fenômeno não detectado imediatamente. Por outro lado, é conveniente alertar que os indicadores não são soluções para todas as dificuldades que envolvem a sustentabilidade, seja na sua avaliação ou na sua operacionalização.

\section{O Modelo Pressão-Estado-Resposta (P-E-R)}

O Modelo PER - "Pressão - Estado - Resposta" - é o marco ordenador mais utilizado para apresentação de estatísticas e indicadores das áreas ambientais e de desenvolvimento sustentável. Foi desenvolvido pela Organização para Cooperação e Desenvolvimento Econômico $(O E C D)$, que regularmente publica seus indicadores nesse formato.

Um marco ordenador pode ser entendido como uma classificação de indicadores em categorias ou, pode estar muito relacionado a uma concepção teórica, específica ao tema estudado, facilitando desse modo a interpretação dos resultados apresentados pelos indicadores (Scandar Neto, 2006).

No caso do modelo PER trata-se da primeira acepção dos termos, a de classificação de indicadores. Martinez, outro pesquisador do tema, além de marcos ordenadores, trabalha com a categoria de marcos referenciais. Essa última expressaria "uma determinada visão dos componentes e inter-relações entre sociedade e natureza (Martinez, 2004). Existiriam quatro tipos de marcos referenciais sobre meio ambiente e desenvolvimento sustentável: marcos simples de componentes ambientais; marco de desenvolvimento sustentável; marco do capital natural (sustentabilidade fraca ou forte) e marcos sistêmico da relação natureza-sociedade. O modelo PER estaria inserido no primeiro marco referencial, que é o mais utilizado na América Latina e que tem como referência a Divisão de Estatísticas das Nações Unidas. Nesse marco "o meio ambiente está constituído por uma série de componentes que podem ser organizados e distinguidos segundo critérios distintos" (Martinez, 2004).

No modelo PER as estatísticas e indicadores relativos a cada tema são divididos em três categorias:

PRESSÃO: Esses indicadores apresentam as pressões que as atividades humanas exercem sobre o meio ambiente. Ex: emissões de poluentes provenientes de fábricas e veículos automotores.

ESTADO: São os indicadores que expressam as condições do meio ambiente tanto em termos quantitativos quanto qualitativos. $O$ objetivo final da política é melhorar esses indicadores. Ex.: índices qualidade do ar.

RESPOSTA: Os indicadores de resposta mostram a reação da sociedade às mudanças das condições ambientais. São as medidas tomadas para diminuir ou anular as pressões ambientais para com isso, melhorar o estado do meio ambiente. Ex.: fiscalização e controle de emissões de poluentes provenientes de fábricas e veículos automotores, que pode ser mensurado na forma de 
Rev. Elet. em Gestão, Educação e Tecnologia Ambiental (e-ISSN: 2236-1170)

número de multas, vistorias, autuações, licenças emitidas, alteração na legislação ambiental, reformulação dos órgãos fiscalizadores etc.

O PSR procura desenvolver indicadores que destacam as ligações entre as atividades humanas, as subsequentes mudanças no estado do ambiente que advêm destas pressões e as respostas da sociedade em relação a estas mudanças. É elaborado após a definição de três tipos de indicadores - pressão, estado e respostas, - que devem refletir o relacionamento entre os efeitos ambientais, suas causas e as medidas necessárias (Oliveira, Lima, Vieira, 2005).

O fluxograma ilustrado na figura 1 demonstra a estrutura conceitual do modelo PSR proposto pela $\mathrm{OECD}$, apresentando uma das possíveis interpretações de como se dá a interação entre as ações humanas e as condições ambientais de um dado sistema.

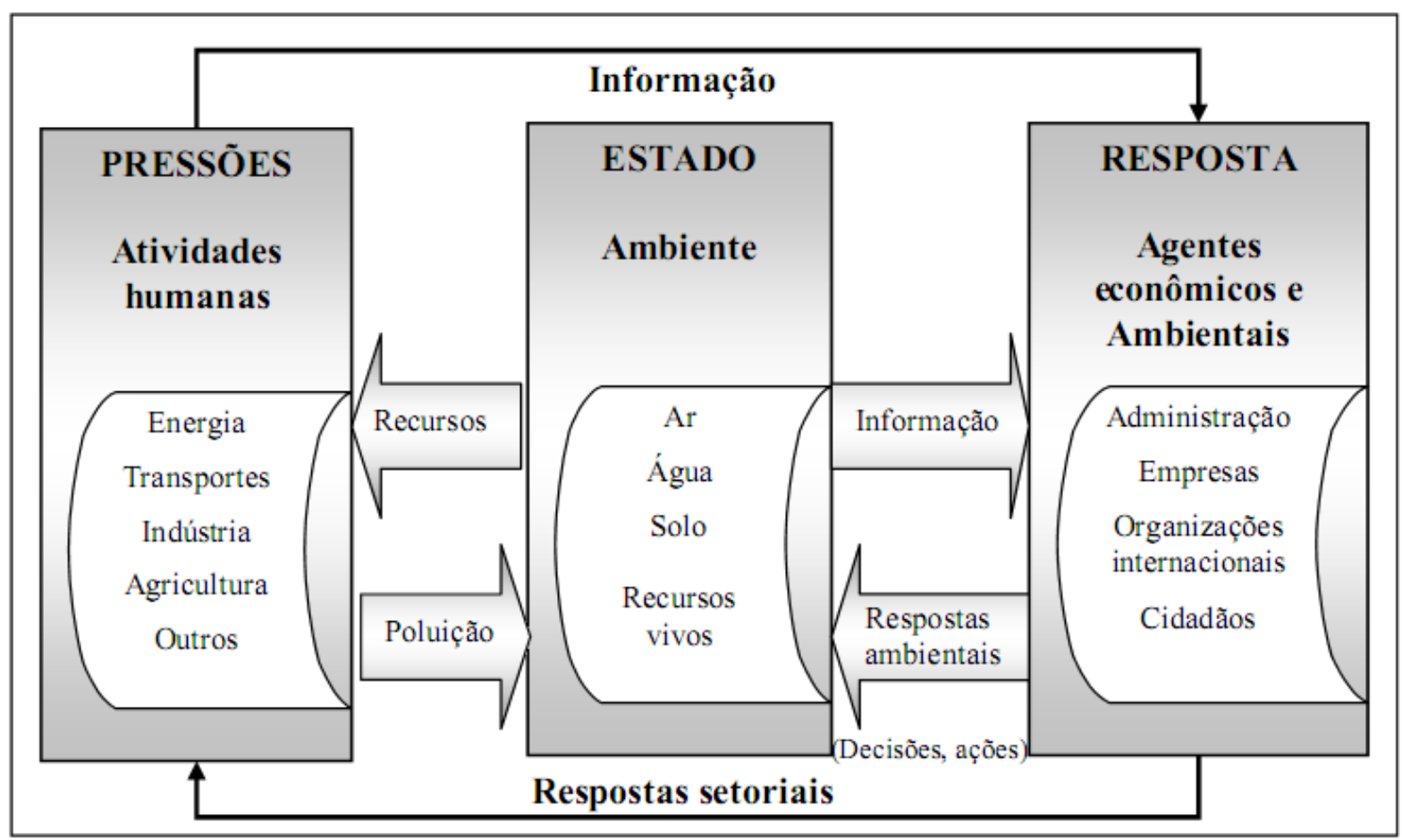

Figura 1. Estrutura conceitual do modelo PSR/PER da OECD Fonte: GOMES et al., 2000, apud TOMASONI, 2006, p. 99.

Em síntese o modelo PER baseia-se na ideia de que, as atividades humanas exercem pressões sobre o meio ambiente e afetam a sua qualidade e a quantidade de recursos naturais (estado); a sociedade responde a estas mudanças, adotando políticas ambientais, econômicas e setoriais, tomando consciência das mudanças ocorridas e a elas adaptando o seu comportamento (resposta da sociedade). Este modelo apresenta a vantagem de evidenciar estes elos e, ajudar os tomadores de decisão e o público a perceber a interdependência entre as questões ambientais e as outras (sem, todavia, esquecer que existem relações mais complexas nos ecossistemas e nas interações meio ambiente-sociedade).

O modelo PER possibilita uma visão conjunta dos vários componentes de um problema ambiental, sendo esta uma grande vantagem que facilita o diagnóstico do problema e a elaboração da respectiva política pública, pois vai além da mera constatação da degradação ambiental e revela seu impacto, suas causas, o que está por trás dessas causas e as ações que estão sendo tomadas para melhorar esse quadro (Carvalho, P., 2007). 
Rev. Elet. em Gestão, Educação e Tecnologia Ambiental (e-ISSN: 2236-1170)

O PER é o modelo mais bem aceito em todo o mundo, sendo o sistema mais usual de estruturação dos indicadores de desenvolvimento sustentável (Santos, 2004) em razão da sua simplicidade e facilidade de uso e pela possibilidade de ser usado em diferentes níveis e atividades humanas, e é considerado um marco de referência para estruturação de indicadores (Cunha, 2002).

No entanto, a principal crítica a esses modelos é que induzem a leitura da existência de uma relação de causalidade linear, o que causa uma simplificação excessiva de uma situação complexa que envolve causalidades múltiplas e interação de fenômenos sociais, econômicos e ambientais. Esse modelo também não estabelecem metas de sustentabilidade a serem alcançadas e como foi concebido originalmente para tratar de indicadores ambientais, nem sempre é adaptável para indicadores de desenvolvimento sustentável (IDS) onde a complexidade é maior (Martinez, 2001).

Ramos et al. (2004) apresentam um levantamento exaustivo de sistemas de indicadores de desempenho ambiental, identificando os respectivos quadros conceituais e os seus objetivos, e conclui que, apesar da grande variedade de sistemas, muitos seguem as mesmas abordagens, sendo na sua maioria adaptações do Modelo Pressão - Estado - Resposta.

\section{Proposta metodológica para aplicação do Modelo P-S-R em Bacias Hidrográficas com análise de vulnerabilidade natural a contaminação}

O termo vulnerabilidade pode ser definido como, a sensibilidade ou suscetibilidade que tem um determinado meio, frente a um impacto, seja ele de origem natural ou antrópica, estando relacionada a erosão do solo, perda de biodiversidade, assoreamento, contaminação do recurso solo - água (Costa et al., 2007). No caso dos aqüíferos, Ribeira (2004) define a vulnerabilidade como uma série de atributos ou características do mesmo, que são o solo, a zona não saturada, os parâmetros hidráulicos do aqüífero e a recarga, que controlam a aptidão do aqüífero para fazer frente a um impacto indeterminado e sua capacidade de auto-restauração.

O Conselho Nacional de Pesquisa norte-americano definiu a vulnerabilidade das águas subterrâneas a um determinado contaminante como a tendência ou probabilidade do contaminante ser encontrado em uma posição específica no sistema de água subterrânea após sua introdução em algum local acima do aquífero mais superior (Leite; Möbus, 2011).

Uma das metodologias mais utilizadas para determinação da vulnerabilidade de aqüíferos, é a metodologia de índices DRASTIC, proposta por Aller et al. (1987). Esses índices de vulnerabilidade correspondem ao somatório ponderado de valores relativos aos seguintes parâmetros hidrogeológicos: profundidade do topo do aqüífero, recarga do aqüífero, material do aqüífero, tipo de solo, topografia, influência da zona vadosa e condutividade hidráulica do aqüífero.

O índice DRASTIC mais elevado significa maior vulnerabilidade. Esses índices são distribuídos em classes de vulnerabilidade e representados na forma de mapa que mostra os fatores hidrogeológicos divididos em subáreas com características intrínsecas representando os diferentes ambientes hidrogeológicos (Cutrim; Campos, 2010).

É importante um estudo conjunto da vulnerabilidade natural e sustentabilidade, a fim de verificar onde as pressões causadas pela ação antrópica se encontram, identificando se as pressões estão localizadas em áreas de vulnerabilidade considerável ou não. No primeiro caso as pressões podem tornar-se riscos potenciais a qualidade ambiental do aquífero, no segundo caso mesmo 
Rev. Elet. em Gestão, Educação e Tecnologia Ambiental (e-ISSN: 2236-1170)

existindo pressões estas pode acabar não gerando impactos ambientais significativos, pois as características naturais do meio podem atenuá-los.

De um modo geral, o termo vulnerabilidade é compreendido como sendo a suscetibilidade do aquífero à contaminação. Segundo Ribeira (2004) a vulnerabilidade natural se costuma calcular e expressar em termos hidrogeológicos, como por exemplo, a profundidade do nível freático, a permeabilidade, etc. Entretanto a vulnerabilidade específica da água subterrânea costuma-se expressar em termos de riscos frente a um determinado impacto.

Uma caracterização aproximada da idéia de risco de poluição das águas subterrâneas consiste na associação e interação da vulnerabilidade natural do aquífero com a carga poluidora aplicada no solo ou em subsuperfície. Isso significa que se pode ter uma situação de alta vulnerabilidade, porém, sem risco de contaminação se não existir carga poluidora significativa, ou vice-versa. A carga poluidora pode ser controlada ou modificada; mas o mesmo não ocorre com a vulnerabilidade natural, que é uma propriedade intrínseca do aqüífero.

A vulnerabilidade significa a maior suscetibilidade de um aqüífero de ser adversamente afetado por uma carga contaminante imposta. Os autores complementam: "É um conceito inverso da capacidade de assimilação de contaminantes de um corpo receptor de água superficial, com a diferença de que os aqüíferos possuem uma cobertura de substratos que proporciona maior proteção" (Foster, Hirata, 1993).

Com relação aos fatores que influenciam a vulnerabilidade dos aqüíferos, Ribeira (2004) comenta que a vulnerabilidade natural de um aqüífero aumenta quanto menor for sua capacidade de atenuação ao impacto e quanto maior for sua acessibilidade.

Aprofundando mais, podem-se discriminar os seguintes grupos de parâmetros que influenciam na determinação da vulnerabilidade: 1) As características geológicas do aquífero: porosidade primária, tipo e grau de fraturação; 2) Os parâmetros hidráulicos do aquífero: sua condutividade hidráulica e sua transmissividade; 3) O regime de recarga do aquífero: tanto em seus aspectos quantitativos como a localização espacial, extensão e magnitude; 4) A existência, continuidade e a espessura da zona saturada e no caso de existir as características hidrológicas e de composição dos níveis suprajacentes do solo.

Osório (2004) destaca que a atividade humana em superfície pode alterar e induzir novos mecanismos de recarga do aquífero, modificando a taxa, a freqüência e a qualidade na recarga de águas subterrâneas. O entendimento desses mecanismos e a correta avaliação de tais modificações são fundamentais para a determinação do risco de contaminação das águas subterrâneas.

É importante lembrar que se existir um aquífero com elevada vulnerabilidade, não significa que este já esteja contaminado, mas sim que esta área é de risco. Sua contaminação ou não vai depender das atividades antrópicas que estão sobre ele localizadas, ou seja, ele pode ser altamente vulnerável, mas não correr nenhum risco de ser contaminado por estar localizado numa área distante de fontes contaminantes, principalmente da presença humana, tais como lixões, cemitérios, distritos industriais entre outros.

As vulnerabilidades estão diretamente associadas às fragilidades intrínsecas da bacia, de um lado, e à superveniência de eventos indesejáveis, tais como eventos extremos e acidentes, em intensidade superior à suportável pelas condições naturais no local considerado. Indicadores de vulnerabilidade são importantes para informar a probabilidade de ocorrência desses eventos, as áreas de risco, as ações preventivas recomendáveis e o esforço de capacitação para minimização dos efeitos de eventos imprevisíveis, mas com histórico de ocorrência na bacia (Maranhão, 2007).

A figura 2 ilustra o uso de metodologias para a determinação da vulnerabilidade, áreas de 
infiltração máxima de água e a aplicação do Modelo P-E-R.

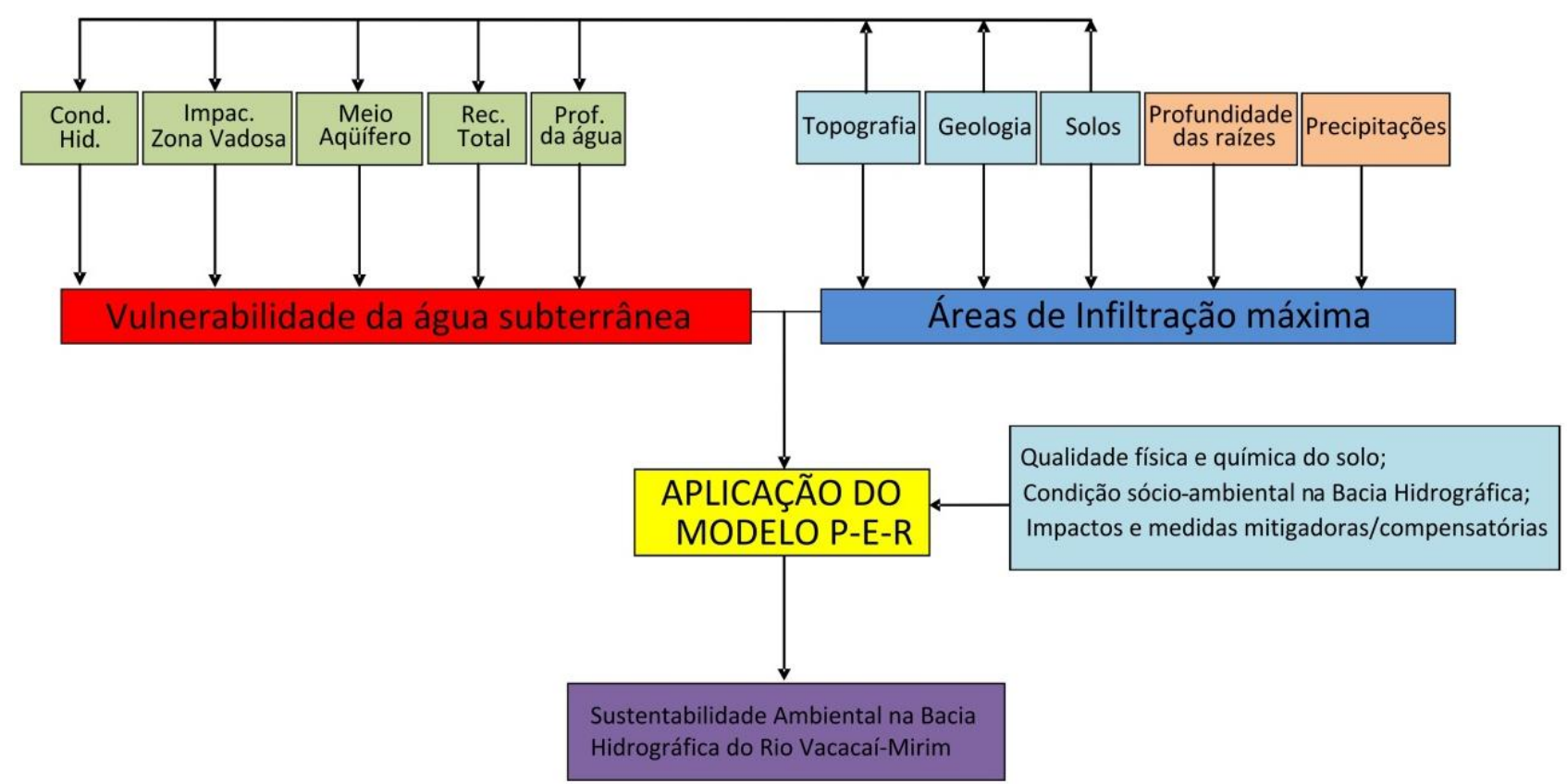

Figura 2. Determinação da vulnerabilidade natural à contaminação da água subterrânea associada ao uso do modelo P-E-R em Bacia Hidrográfica.

As pressões são identificadas através do levantamento dos usos do solo na Bacia Hidrográfica, neste caso a ênfase dada ao modelo P-E-R é a qualidade física e químicos do solo, sendo avaliada a ocorrência metais pesados, densidade do solo, porosidade do solo, condutividade hidráulica saturada, textura do solo e densidade de partícula.

As respostas podem ser obtidas através da análise de Atas do Comitê de Gerenciamento de Bacias Hidrográficas, onde podem existir relatos de ações da sociedade para minimizar, mitigar ou compensar os impactos ambientais decorrentes das atividades desenvolvidas na área da Bacia.

Dessa forma identificando-se as Pressões (atividades desenvolvidas na Bacia Hidrográfica), - Estado do ambiente decorrente das pressões e as Respostas dadas pela sociedade aos problemas ambientais tem-se um instrumento capaz de nortear as tomadas de decisão no âmbito do gerenciamento dos Recursos Hídricos que não só apresenta os impactos ambientais na área estudada, mas também identifica sua origem, o quantifica, e levanta as medidas adotadas até o momento de sua avaliação possibilitando assim intervenções mais precisas principalmente pelos órgãos gestores da qualidade ambiental.

\section{CONCLUSÃO}

Com base no objetivo proposto, pode-se concluir que os indicadores de sustentabilidade aliados a aplicação de metodologias de análise de vulnerabilidade são capazes de identificar quantificar os impactos ambientais em bacias hidrográficas, especialmente em suas áreas mais susceptíveis a eventos danosos ao meio ambiente. Dessa forma é possível também identificar as medidas tomadas até o momento da avaliação e incrementá-las e/ou modificá-las tornando-as 
mais eficientes podendo estas serem direcionadas as áreas onde realmente se fazem necessárias, demonstrando assim a importância de tais instrumentos no gerenciamento de recursos naturais.

\section{REFERÊNCIAS BIBLIOGRÁFICAS}

ALLER, L.; LEHR, J. H.; PETTY, R. DRASTIC: a standardized system for evaluating groundwater pollution potencial using hydrogeologic settings. [Documento online]. Disponível em: < http://info.ngwa.org/gwol/pdf/860138698.PDF>. Acesso em: 16/11/2011.

BELTRAME, A. V. Diagnóstico do meio físico de bacias hidrográficas: modelo e aplicação. Florianópolis: UFSC, 1994.

BOUNI, C. Indicateurs de développement durable: I"enjeu d"organiser une information hétérogène pour préparer une décision multicritère. In: COLLOQUE INTERNATIONAL, 1996, Porto. Anais... Porto: Cl, 1996.

CARVALHO, P. G. M. de.; BARCELLOS, F. C.; MOREIRA, C. G. (2007) Políticas públicas para meio ambiente na visão do gestor ambiental- Uma aplicação do modelo PER para o Semi-Árido. In: VII ENCONTRO DA SOCIEDADE BRASILEIRA DE ECONOMIA ECOLÓGICA, Fortaleza, 2007. Anais... Recife: VII ESBEE, 2007.

CARVALHO, J. R. M. de; CURI, W. W. F; CARVALHO, E. K. M. de A, CURI, R. C. Proposta e validação de indicadores hidroambientais para bacias hidrográficas: estudo de caso na sub-bacia do alto curso do Rio Paraíba, PB. Revista Sociedade e Natureza, Uberlândia, v. 23, n. 2, 2011.

CLAIN, N. Les indicateurs de développement durable en agriculture, aspects écologiques et environnementaux. Paris: Université de Paris, 1997.

COSTA, T. C. E. C.; UZEDA, M. C.; FIDALGO, E. C. C.; LUMBRERAS, J. F.; ZARONI, M. J.; NAIME, U. J.; GUIMARAES, S. P. Vulnerabilidade ambiental em sub-bacias hidrográficas do Estado do Rio de Janeiro por meio de integração temática da perda desolo (USLE), variáveis morfométricas e o uso/cobertura da terra. In: XII CONGRESSO BRASILEIRO DE SENSORIAMENTO REMOTO, 2007, Florianópolis. Anais... Florianópolis: XIII SBSR, 2007.

CUNHA, F. L. S. J. O uso de indicadores de sustentabilidade na avaliação dos impactos das atividades econômicas e na tomada de decisões. In: ENCONTRO DE ECONOMIA PARANAENSE, 2002, Maringá. Anais... EEP: Maringá, 2002.

CUTRIM, A. O.; CAMPOS, J. E. G. Aplicação dos Métodos Drastic e Posh para a Determinação da Vulnerabilidade e Perigo à Contaminação do Aquífero Furnas na Cidade de Rondonópolis-MT. Revista Brasileira de Recursos Hídricos, São Paulo, v. 15, n. 2, p. 127 - 142, 2010.

FOSTER, S. S. D.; HIRATA, R. C. A. Determinação do risco de contaminação das águas subterrâneas: um método baseado em dados existentes. São Paulo: Instituto Geológico. São Paulo, 1993.

FRANCA, L. P. Indicadores ambientais urbanos: revisão da literatura. ISER/ IBAM: Parceria, 2001.

FREUDENBERG, M. Composite indicators of country performance: a critical assessment. Paris: OECD, 2003.

FREYENBERG, S.; JANKE, R.; NORMAN, D. Indicators of Sustainability in Whole- Farm Planning: literature review. Kansas Sustainable Agriculture Series, Manhattan, v.2, 1997.

GOMES, P. R; MALHEIROS, T. F. Proposta de análise de indicadores ambientais para apoio na discussão da sustentabilidade. Revista Brasileira de Gestão e Desenvolvimento Regional, Taubaté, v. 8, n. 2, p. 151-169, 2012.

HAMMOND, A. Environmental indicators: a systematic approach to measuring and reporting an environmental policy performance in the context of sustainable development. Washington: World Resource Institute, 1995. 
JORGENSEN, S.E. Introduction. In: JORGENSEN, S.E.; COSTANZA, R.; XU, F.L. (Eds.). Handbook of ecological indicators for assessment of ecosystem health. New York: CRC Press Taylor \& Francis Group, 2005.

LEITE, C. E. S.; MÖBUS, G. Vulnerabilidade natural à contaminação dos aqüíferos da região de rochas sedimentares da bacia do rio mundaú / Ceará. In: X congresso Brasileiro de Águas Subterrâneas, São Paulo, 1998. Anais... São Paulo: X CBAS, 1998.

MACDONALD, S. Benchmarking and Sustainability Indicators. Rushmoor Borough Council, 1996. [Documento online]. Disponível em: <http://www.rushmoor.gov.uk/gr916.htm>. Acesso em 06/11/2011.

MARANHÃO, N. Sistema de Indicadores para Planejamento e Gestão dos Recursos Hídricos de Bacias Hidrográficas. Rio de Janeiro. 2007. 397 p. Tese de Doutorado - Universidade Federal do Rio de Janeiro, Rio de Janeiro, 2007.

MARTINEZ, R. Indicadores de Sostenibilidad Ambiental y de Desarrollo Sostenible: Estado del Arte y Perspectivas. Série Manuales, $n^{\circ} 16$, Santiago de Chile: CEPAL, 2001. [documento online]. Disponível em: <http://www.eclac.cl/publicaciones/xml/8/9708/Icl1607e_ind.pdf>. Acesso em 17/10/2012.

MARTINEZ, R. Q. Indicadores de sustentabilidade: avanços e desafios para a América Latina. São Paulo: Unicamp \& Imprensa Oficial, 2004.

MARZALL, K.; ALMEIDA, J. Parâmetros e indicadores de sustentabilidade na agricultura: limites, potencialidades e significado no contexto do desenvolvimento rural. Extensão Rural, Santa Maria, n. 5: p. 25-38, 1998.

MARZAL, K.; ALMEIDA, J. Indicadores de sustentabilidade para agroecossistemas: Estado da arte, limites e potencialidades de uma nova ferramenta para avaliar o desenvolvimento sustentável. Cadernos de Ciência \& Tecnologia, Brasília, v.17, n.1, p.41-59, jan./abr., 2000.

OLIVEIRA, R. E. S.; LIMA, M. M. C. L.; VIEIRA, J. M. P. An indicator system for surface water quality in river basins. In: INTER-CELTIC COLLOQUIM ON HYDROLOGY AND MANAGEMENT OF WATER RESOURCES, 2004, Guimarães. Anais... Guimarães: ICHMWR, 2005.

OSÓRIO, Q. Da. S. Vulnerabilidade Natural de Aquíferos e Potencial de Poluição das Águas Subterrâneas. 2004. 139f. Dissertação de Mestrado - Universidade Federal de Santa Maria, Santa Maria, 2004.

RAMOS, T. B.; CAEIRO, S.; MELO, J. J. Environmental Indicator Frameworks to Design and Assess Environmental Monitoring Programs, Impact Assessment and Project Appraisal. United Kingdom, n.22: p.47-62, 2004.

RIBEIRA, F. Calidad, contaminación y protección de acuíferos. In: III CURSO HISPANOAMERICANO DE HIDROLOGÍA SUBTERRÁNEA, 2004, Montevidéu, Anais... Montevidéu: IIICHHS, 2004.

SANTOS, R. F. Dos. Planejamento Ambiental: teoria e prática. São Paulo: Oficina de textos, 2004.

SCANDAR NETO, W. J. Indicadores de desenvolvimento sustentável no Brasil. In: SEMINÁRIO FLUMINENSE DE INDICADORES, Rio de Janeiro, 2004. Anais... Rio de Janeiro: Fundação CIDE, 2004.

TOMASONI, M. A. Contribuição ao estudo de indicadores ambientais. Rio de Janeiro, Geonordeste, 2006.

VAN BELLEN, H. M. Indicadores de Sustentabilidade: Uma Análise Comparativa. Rio de Janeiro: FGV, 2006. 